\title{
Implementation and performance evaluation of an FBMC-based PMR system for broadband PPDR applications
}

\author{
Oriol Font-Bach, Nikolaos Bartzoudis, David López, Miquel Payaró \\ Centre Tecnològic de Telecomunicacions de Catalunya (CTTC), Castelldefels, Barcelona, Spain \\ Email: \{oriol.font, nikolaos.bartzoudis, david.lopez, miquel.payaro\}@cttc.cat
}

\begin{abstract}
In this paper, an experimental performance evaluation is carried out within a Professional Mobile Radio / Public Protection and Disaster Relief (PMR/PPDR) communication scenario that features two related key enablers for the evolution towards broadband transmission in those systems: (i) the use of post-OFDM modulations (ii) an efficient use of the spectrum via spectrum sharing among the legacy narrowband tranmissions and the newly proposed broadband transmission. The real-time experimental laboratory setup was assembled to operate in conditions as realistic as possible by combining hardware prototypes for the broadband transmitters, commercial PMR terminals and a radio channel emulator (providing mobile channels conditions based on the Extended Pedestrian A specifications). The experimental results show that co-existence in a shared spectrum scenario is possible and that the performance degradation is kept at a low level, as long as the broadband transmission is making use of spectrally agile post-OFDM modulations such as filterbank multicarrier (FBMC).
\end{abstract}

\section{INTRODUCTION}

Professional Mobile Radio (PMR) systems for Public Protection and Disaster Relief (PPDR) services and applications are fundamentally used for low-rate data transmission like voice communications. Recently, there has been a trend to upgrade the PMR systems to support high-rate applications and broadband services. To upgrade the PMR/PPDR networks (TETRAPOL, TEDS, etc.) towards supporting broadband data communications services, additional capacity is required, which can be achieved via two complementary alternatives; i) by obtaining new bandwidths for PMR data services, and ii) by fitting a new broadband data service within available fragmented spectrum devoted to PMR systems (e.g., in reserved bands at: $380-400 \mathrm{MHz}, 410-430 \mathrm{MHz}$, or $450-470 \mathrm{MHz}$ ).

The second option is of particular interest for spectrum owners, infrastructure providers and site holders since the introduction of new broadband data services within the current frequency allocation, in coexistence with deployed PMR/PPDR systems (legacy) is an efficient way to optimize the total cost of ownership (TCO). In this repsect, it is important to mention that extensive Long Term Evolution (LTE) trials with pilot networks were conducted towards this end to explore the feasibility of adding mobile broadband capabilities to

This work was partially supported by the Generalitat de Catalunya under grant 2014 SGR 1551; and by the European Commission under projects EMPhAtiC (GA 318362) and NEWCOM\# (GA 318306). existing Tetrapol and TETRA networks [1]. The objective of the pilots was to validate how leveraging existing sites and frequency bands for the new technology affects coverage and capacity. One of the findings of these studies was that the premium radio frequency propagation characteristics of lower frequency bands can indeed help to increase cell coverage, a crucial aspect when it comes to providing services in rural and sparsely populated areas. $1.4 \mathrm{MHz}$ channels were considered to be a good way to start, with $5 \mathrm{MHz}$ being the final target for the projected delivery of broadband services, a fact that will eventually require a frequency re-farming of the PMR systems at the $400 \mathrm{MHz}$ band. In this framework, the use of LTE technology is planned for broadband PPDR implementations.

The goal of this paper is to show the suitability of filter bank multi carrier (FBMC) modulations as a future technology substitute or evolution of the LTE Physical layer based on orthogonal frequency division multiplexing (OFDM). Indeed, on top of the advantages offered by existing solutions (e.g., increase coverage, reduce TCO) that aim to integrate broadband LTE services at the available fragmented spectrum of the PMR bands [2], the developed FBMC system can offer a nearly optimal spectral contention and spectral efficiency avoiding at the same time interference at adjacent primary transmissions. The authors in [3] have showed through simulation results that FBMC-broadband system could be considered as a potential candidate to be deployed in the PMR band. Other advantages of FBMC over OFDM have also been reported in [4].

The work presented in this paper was conducted in the framework of the project EMPhAtiC (funded by the European Commission) and demonstrates the coexistence of narrowband PMR systems operating at $400 \mathrm{MHz}$ with a broadband LTElike (but FBMC-based) communication system. As it will be shown herein, the benefits of the LTE-like FBMC system versus its LTE-OFDM counterpart were proved at a practical level by developing a real-time hardware demonstrator.

\section{TARGETED SCENARIO AND LAB SET-UP}

Due to the high complexity involved with the experimental validation of communication scenarios with spectrum sharing and coexistence, a rather simple, yet highly representative operating scenario for PMR/PPDR has been considered based on the real-time DL communication system that was built and demonstrated as part of the EC-funded project EMPhAtiC [5]. 


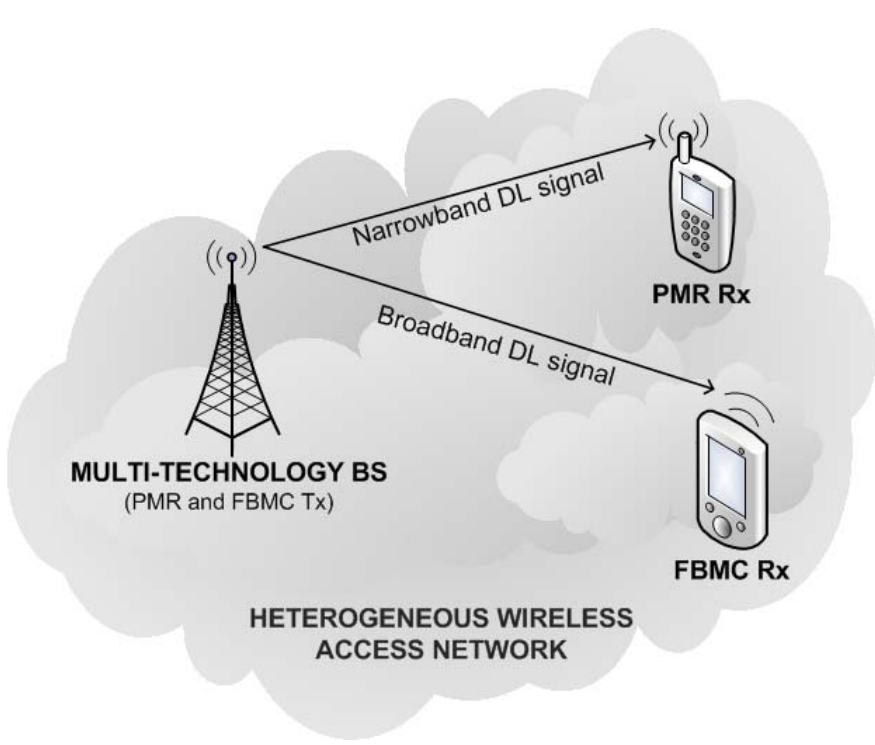

Fig. 1. High level description of the implemented scenario.

The end use scenario features a primary narrowband PMR transmission coexisting with a LTE-like opportunistic FBMCbased broadband transmission. Both systems are sharing the same frequency band and make use of a point-to-point DL communication link. A graphical representation of the scenario targeted in this work can be found in Fig. 1. In this kind of scenarios, it is a hard requirement to dynamically adapt the FBMC signal so as not to interfere with the primary transmission. This is made feasible by deactivating subcarriers in the broadband transmission. The FBMC DL system is also capable of applying dynamic subacarrier allocation serving likewise cognitive radio use cases, but this feature is not covered in this paper.

In the following subsections, we present a detailed description of the different lab set-ups that have been assembled in order to evaluate the performance of the communication scenario depicted in Fig. 1. In particular, the three lab set-ups present an increasing complexity in order to capture (also with an increasing level of realism) the different aspects that impact the achievable performance: i) cabled set-up and off-line baseband operation, ii) emulated wireless channel and realtime operation, and iii) over-the-air and real-time operation.

\section{A. Cabled set-up}

As a first step towards the experimental evaluation of the scenario described in Section II, we deployed a baseline hardware setup with the aim to observe the performance of a legacy narrowband PMR system in a coexistence scenario, both with cyclic prefix (CP)-OFDM and FBMC transmissions. More specifically, a single Tetrapol transmitter and receiver pair (using hardware provided by Airbus) did constitute the primary communication, whereas a single CP-OFDM/FBMC transmitter and receiver pair provided the opportunistic secondary broadband transmission.

Both Tetrapol and broadband signals were generated off-

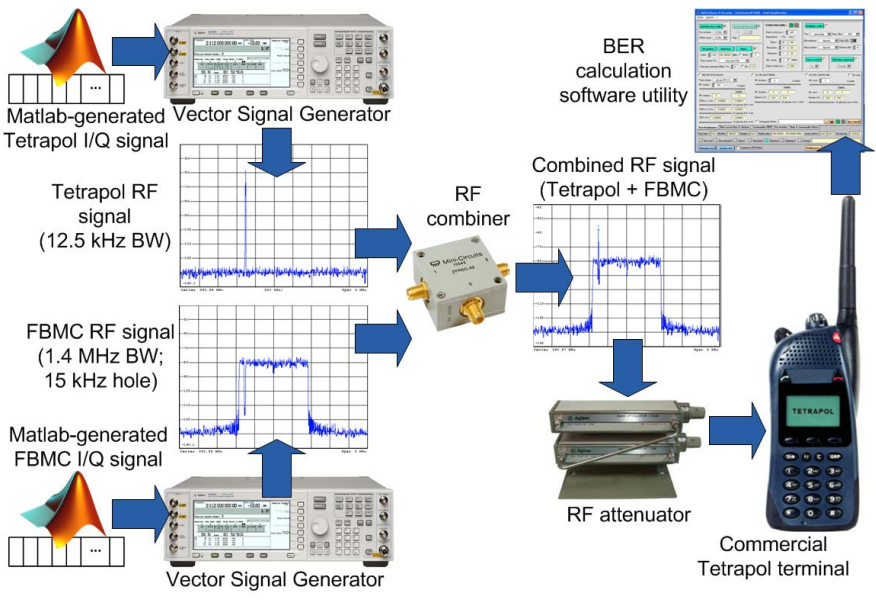

Fig. 2. Basic hardware setup with cabled channel.

line in Matlab and the resulting I/Q data-vectors were then uploaded onto two different Vector Signal Generators (VSGs), which provided two real-world RF signals. The broadband transmission (both for CP-OFDM and FBMC schemes) had $1.4 \mathrm{MHz}$ of signal BW with up to 72 active $15 \mathrm{kHz}$ subcarriers (following the LTE standard). Out of those 72 subcarriers, only 70 were actually used for transmission by the broadband system; the unallocated $30 \mathrm{kHz}$ were then used by the Tetrapol transmission, which actually required $12.5 \mathrm{KHz}$, as it can be observed in Fig. 2. The two resulting RF signals were then combined, providing the single RF signal, which then passed through a series of RF attenuators to ensure that the received signal power was as desired (an ideal channel situation is thus considered in this cabled-equipment setup). The resulting RF signal was then fed to a commercial Tetrapol terminal, which was set to "test mode". A proprietary software application (also provided by Airbus) finally allowed computing the BER observed at the terminal.

The previously described lab setup was used to experimentally analyse the performance observed in the Tetrapol terminal for different relative subcarrier power levels between the primary and secondary transmissions. That is, various system configurations were considered, ranging from an ideal situation where (at reception side) the Tetrapol signal was $21 \mathrm{~dB}$ above the broadband one, to an extreme case where the primary signal was almost $27 \mathrm{~dB}$ under the interfering opportunistic transmission (Fig. 3). For each considered relative subcarrier power level value, the BER value was computed by averaging 10.000 frames.

Fig. 4 shows the BER performance observed at the Tetrapol terminal when coexisting with opportunistic signal transmissions on the same RF band, both for broadband CP-OFDM and FBMC transmission schemes. It is important to note that the Tetrapol terminal stopped working (i.e., could not synchronize) whenever the received power for the PMR signal was less than $5 \mathrm{~dB}$ above of that of the OFDM. Until reaching that extreme situation, the Tetrapol terminal is still capable of providing a good quality of voice service (i.e., BER below $1 \%$ ). 


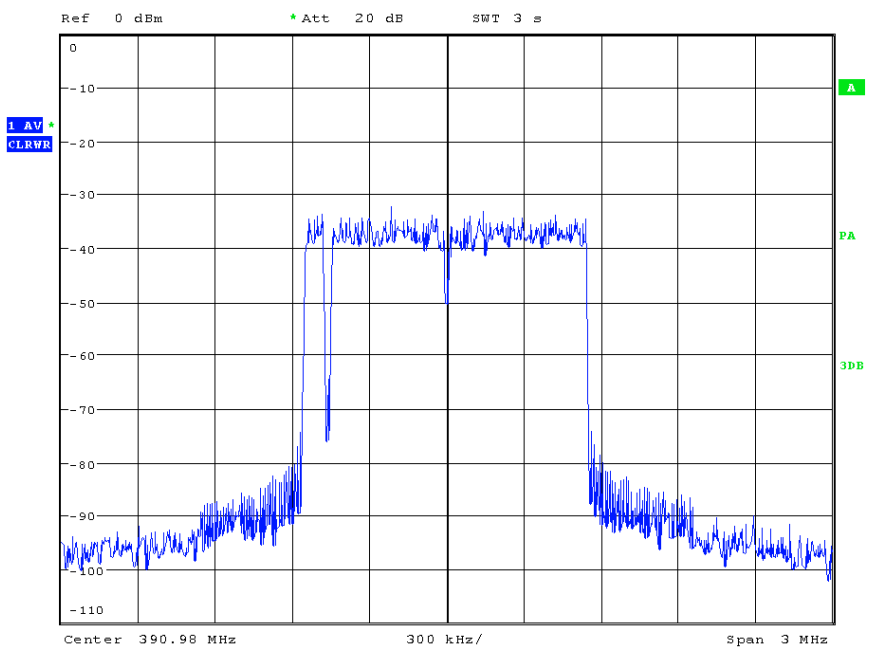

Fig. 3. Example of a considered received-signal power relation in the coexistence scenario featuring a narrowband Tetrapol system and an opportunistic broadband transmission. The Tetrapol signal power is $26.9 \mathrm{~dB}$ below the subcarrier level of the broadband FBMC transmission. In the figure, the Tetrapol spectrum is almost hidden in the spectrum hole that the broadband transmission leaves unused on the left hand side of the spectrum.

Regarding the opportunistic broadband FBMC transmissions, the Tetrapol system could render a good quality voicecall up to a situation where the received PMR signal power was $18.9 \mathrm{~dB}$ below of that of the FBMC. That is, the range of signal power-ratios under which the PMR system can operate normally in a coexistence scenario with FBMC is extended by $23.9 \mathrm{~dB}$ (with respect to a coexistence with OFDM transmissions). Observe that, even when the PMR signal power is up to $23.9 \mathrm{~dB}$ below that of the FBMC one, the voice quality of the PMR system is maintained despite the BER degradation, which reaches a lower bound of $2.5 \%$.

Once this preliminary cabled set-up had been properly tested and validated, the next step is to move closer to actual system operation in the field by adding real-time operation features and emulated wireless channels with the aim to mimic the behaviour of actual channels encountered in practice.

\section{B. Complete hardware set-up}

The setup described in the previous subsection was upgraded by replacing the VSGs with a custom real-time SoCbased FBMC transmitter and by including a real-time multichannel emulator in order to evaluate the performance of both Tetrapol and FBMC systems under realistic mobile channel conditions. This second real-time hardware setup (see Fig. 5) was configured as follows:

- A VSG was used to cyclically play back a known Tetrapol I/Q frame sequence (provided by Airbus), emulating likewise the real-time narrowband primary transmission. This signal enabled the computation of the BER at the PMR terminal. The DAC and upconversion modules of the VSG allowed to synthesize and fine-tune the resulting RF signal (centered at $382.53 \mathrm{MHz}$ ).

- On the FBMC transmission side a prototyping platform

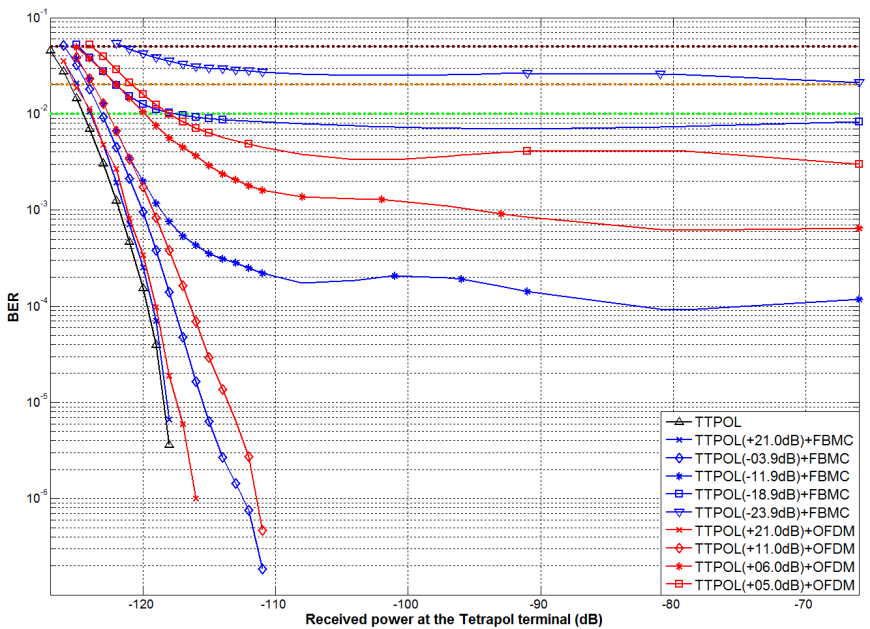

Fig. 4. BER versus received signal power in the cabled set-up for different power ratios between the primary and secondary transmissions and also for different technologies used for the secondary transmission (FBMC and CPOFDM).

which combines the Xilinx ZC706 board and the Analog Devices AD-FMCOMMS3-EBZ RF transceiver board, was utilized. The ZC706 board features the XC7Z045 FPGA-based SoC device which embeds a dual $\mathrm{ARM}^{\circledR}$ Cortex $^{\mathrm{TM}}$-A9 GPP and large programmable logic area, whereas the RF frontend features a versatile AD9361 RF IC from Analog Devices (2x2 MIMO capable, $70 \mathrm{MHz}$ to $6 \mathrm{GHz}$ operation, $56 \mathrm{MHz}$ analog $\mathrm{BW}$ ), which, for the needs of the test setup, provided an RF signal centered at $382.88 \mathrm{GHz}$. See [6] for further implementation details.

- Both RF signals were fed to the Elektrobit Propsim C8 multi-channel emulator which provided realistic channel propagation conditions. The 3GPP Extended Pedestrian A (EPA) channel model at $3 \mathrm{~km} / \mathrm{h}$ (Table I) was used for the experimental validation of this hardware setup.

- The signals at the output of the channel emulator were driven through a series of RF step attenuators to control the power ratio between the two coexisting transmissions.

- The power-adjusted RF signals were combined into a single RF signal. An in-house RF filter was additionally used to eliminate undesired out-of-band components. An RF splitter was added to provide two exact replicas of the combined RF signals, which were then fed to the FBMC and PMR receivers.

- A M9620SG2 Tetrapol terminal device configured in test mode acted as the PMR receiver for the primary transmission. The PMR terminal was connected with a laptop PC through a custom cable connection, where a software application from

\begin{tabular}{|l|c|c|c|c|c|c|c|}
\hline Tap & 1 & 2 & 3 & 4 & 5 & 6 & 7 \\
\hline \hline Relative delay $(\mathrm{ns})$ & 0 & 30 & 70 & 90 & 110 & 190 & 410 \\
\hline Average power (dBm) & 0 & -1 & -2 & -3 & -8 & -17.2 & -20.8 \\
\hline
\end{tabular}

RELATIVE DELAY AND AVERAGE POWER OF THE CHANNEL TAPS. EACH CHANNEL TAPS FOLLOWS A RAYLEIGH DISTRIBUTION. 


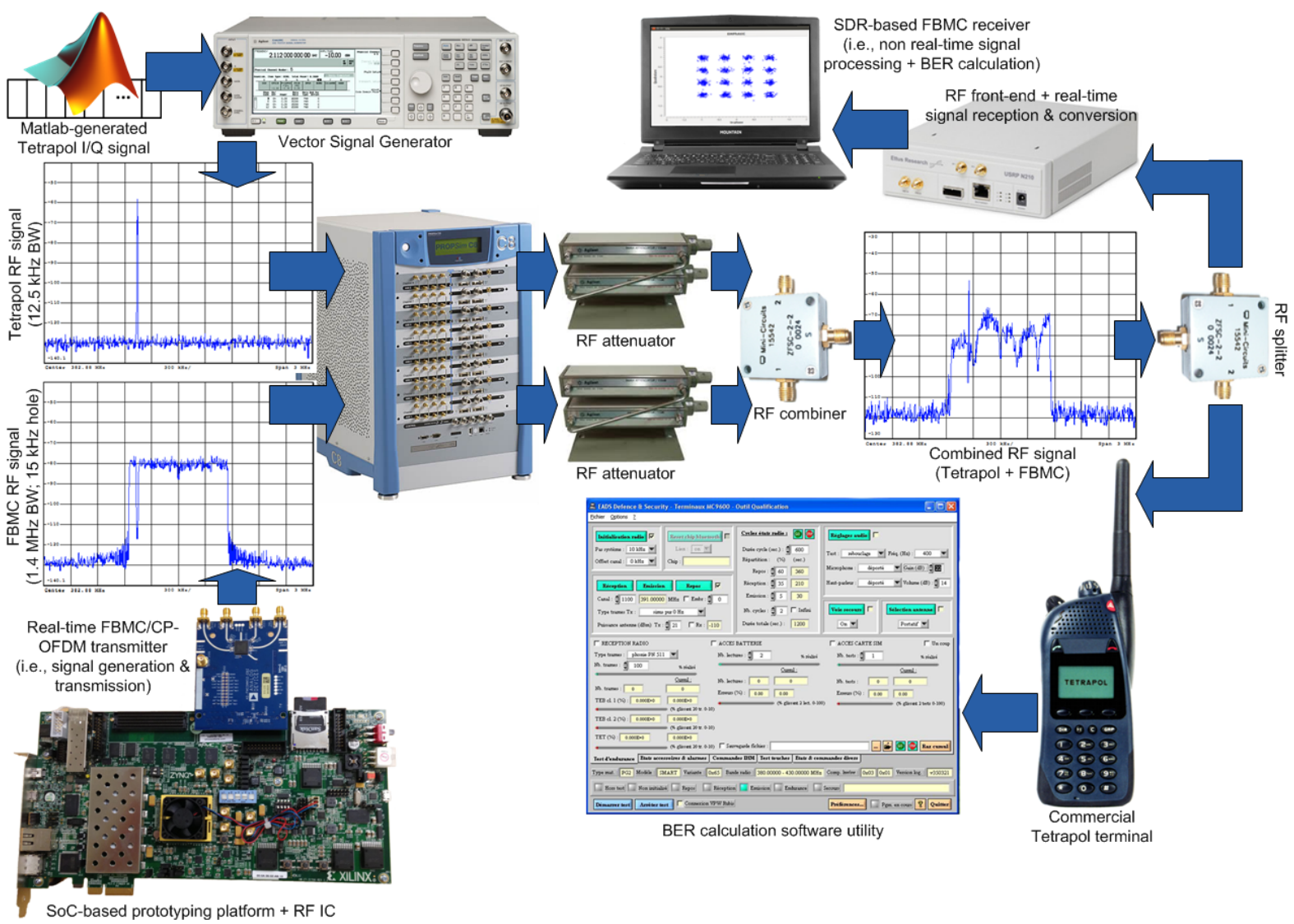

Fig. 5. Hardware setup assembled in the lab.

Airbus enalbed the calculation of the BER averaging a given number of frames.

- The FBMC receiver used the Ettus Research USRP N210 device (RF signal down conversion, signal acquisition and digital down conversion) which provided the complex baseband samples to a laptop PC through a dedicated Gigabit Ethernet (GigE) interface. The SDR-based FBMC receiver was implemented at the same laptop allowing as well the computation and storage of the frame-based BER metrics. For more details on this implementation see [6].

Fig. 6 illustrates the performance observed at the Tetrapol terminal under the EPA channel model, featuring a mobile speed of $3 \mathrm{~km} / \mathrm{h}$, when coexisting with both broadband FBMC and CP-OFDM transmissions. The experimental measurement campaign proves that the demonstrator has successfully achieved its proof-of-concept goals, allowing to quantize the gains brought by the use of FBMC systems in the considered scenario. It is important to highlight the coherence of the results observed in Fig. 6 where the real-time transmitter and the channel emulator were used, with those obtained in Fig. 4 for the cabled set-up and the offline transmissions. This observation allows us to validate the results obtained in this section and consider the results in the previous section as a necessary step towards actual system implementation after simulation results have been obtained.
As shown in Fig. 6, under a mobile EPA channel the FBMC system is able to provide a gain of roughly $25 \mathrm{~dB}$ when compared to the CP-OFDM scheme, in terms of performance observed at the Tetrapol terminal, as well as in terms of

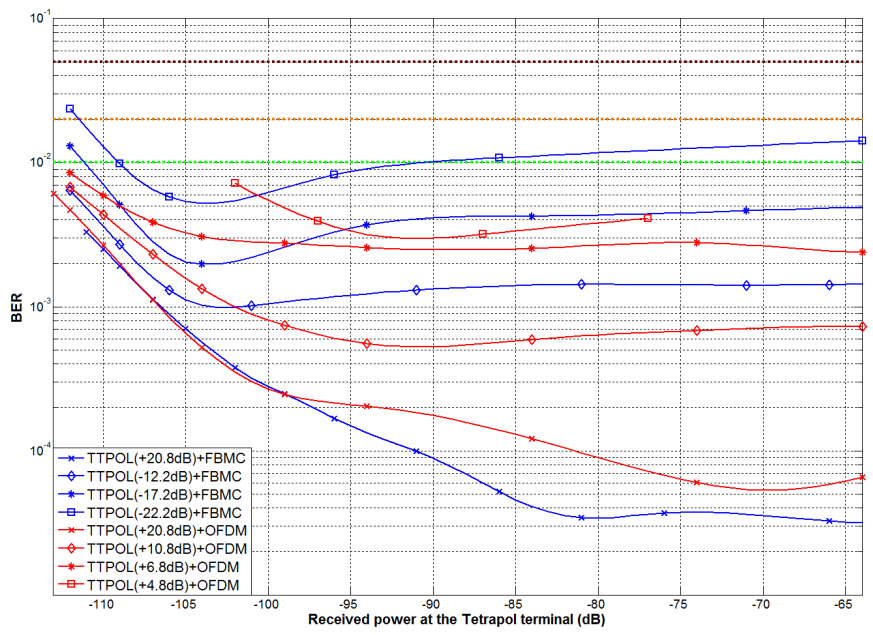

Fig. 6. BER versus received signal power under the EPA channel model for different power ratios between the primary and secondary transmissions and also for different technologies used for the secondary transmission (FBMC and $\mathrm{CP}-\mathrm{OFDM}$ ). 


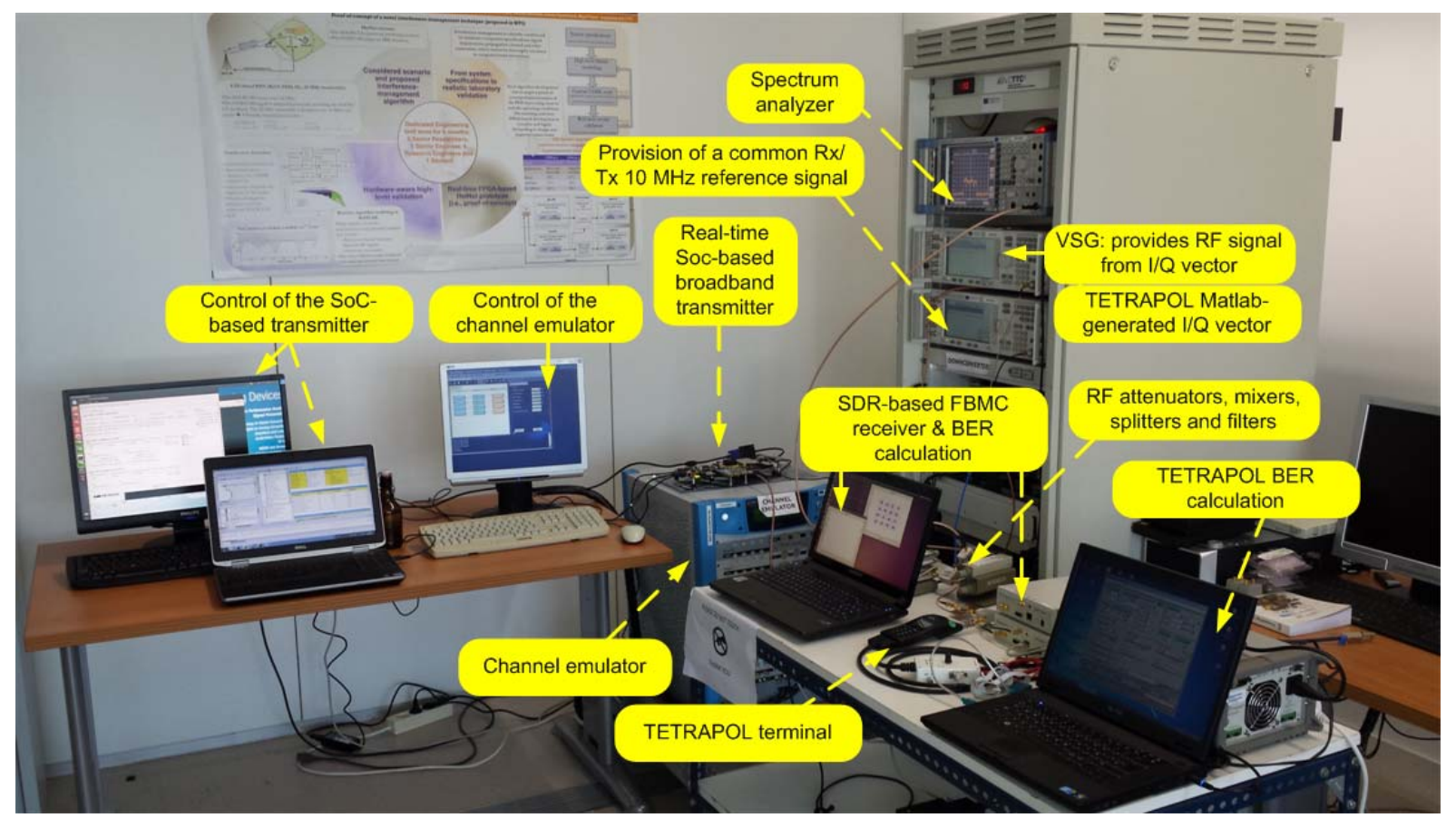

Fig. 7. Photo of the lab set-up.

sensitivity to baseband interferences. Moreover, the second hardware setup of the demonstrator considers stringent operating conditions such as reduced frequency guard-band between the two coexisting transmissions. Therefore, higher gains could be expected at the cost of a less efficient spectrum reuse when considering, for instance, wider holes in the broadband signal.

A photo of the complete hardware setup comprising the real-time testbed is shown in Fig. 7.

\section{Over-the-air qualitative trials}

A third setup was also assembled with two Tetrapol terminals communicating over-the-air. Appropriately selected antennas were used in the real-time SoC-based transmitter. The over-the-air tests aimed at recreating the operation conditions introduced in the first hardware setup (Section II-A), where the PMR signal power was slightly above that of the broadband one. The precise power-ratio depended on the relative location of the two Tetrapol terminals and also accounting that to the broadband transmitter. Moreover, the received signal power was close to the sensibility limits of the terminal (i.e., a $50 \mathrm{~dB}$ attenuator was placed between the antenna and its connector in the Tetrapol terminal). In that situation, the opportunistic CP-OFDM transmission was strongly impairing the direct mode / ad-hoc voice communication, which was only possible if the two Tetrapol terminals were close one to each other (i.e., less than two meters) and in line of sight. On the other hand, a perfect voice reception was observed under the same conditions when switching the broadband signal to the proposed FBMC scheme, allowing the two terminals to freely move inside the laboratory.

\section{CONCLUSION}

In the experimental evaluation reported in this paper, we have compared the achieved performance in terms of BER versus received signal power of a PMR/PPDR system that featured coexistence in a shared spectrum scenario. In particular, we have compared FBMC and OFDM as candidates for the physical layer of the secondary transmission and we have shown the superior performance that yields the use of FBMC. The experimental set-up assembled in this work will be used for further research work by endowing it with spectrum sensing and dynamic spectrum allocation capabilities so that more advanced features can also validated in such a realistic lab environment.

\section{REFERENCES}

[1] P. Mège, L. Martinod, A. Cipriano, "Reconfigurable Radio Systems potentialities for the civil security domain," in the White Paper EMPhAtiC: Progress towards standardization and regulation. Available on-line: http://www.ictemphatic.eu/images/deliverables/white_paper_standardization.pdf

[2] EVERCOR - The Integrated LTE 400 PMR Solution. Webpage accessed May 2015: http://www.alcatel-lucent.com/public-safety/evercor-theintegrated-lte-400-pmr-solution

[3] Y. Medjahdi, D. Le Ruyet, F. Bader, L. Martinod, "Integrating LTE broadband system in PMR band: OFDM vs. FBMC coexistence capabilities and performances," in the Proceedings of the 2014 11th International Symposium on Wireless Communications Systems (ISWCS), pp. 643 648, 26 - 29 Aug. 2014, Barcelona, Spain.

[4] M. Payaró, A. Pascual-Iserte, M. Nájar, "Performance comparison between FBMC and OFDM in MIMO systems under channel uncertainty," in the Proceeding of the 16th European Wireless Conference, pp. 10231030, Apr 2010, Lucca, Italy.

[5] EMPhAtiC D9.4, "Evaluation of the implemented communication system", February 2015.

[6] EMPhAtiC D9.1, "Definition and specification of hardware demonstrator and software simulator", March 2014. 